\title{
Coverillustration
}

\section{An eye for the ladies}

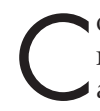
opepods ("oar-footed") are the most numerous of the metazoa and play an enormously important part in the food chain. In the colder waters closer the poles their biomass is so extraordinary that they sustain all manner of invertebrates and vertebrates from the lowly worms up to the baleen whales. Without these diminutive creatures our oceanic food chain would collapse, for these are primary consumers of microscopic bacteria and protists that are the base of the food pyramid. Copepods, members of the phylum Crustacea, are generally no larger than $1 \mathrm{~mm}$ long, but a few species may be somewhat larger. They can be found in all aquatic ecosystems throughout the world, including many cave systems or even leaf litter. Crustacea, like insects, are arthropods, but have more diversity of eye types than insects or, for that matter, any other invertebrate group. Within the Crustacea, there are single chambered eyes, all forms of compound eyes, and some forms of optical creativity seen in no other species. Certain species of pontellids, a family of copepods, have such extraordinary and unusual eyes that the optics remain a mystery to this day.

Pontella scutifer is a free living marine copepod found irregularly throughout the world's oceans, although truly epipelagic and usually living well offshore. This species has three eyes and one of them is quite peculiar, as can be seen on this month's cover. There are two simple eyes on the dorsal aspect of the cephalosome, or carapace equivalent (upper cover image). These are found in both males and females and probably have very poor acuity, used basically to determine light, dark and, perhaps, movement. In this simple eye, there is a lens and a few photoreceptors found directly beneath and almost in direct contact with the lens. The lens material probably has a very high index of refraction and even then probably cannot focus an image on the photoreceptive elements. It doesn't really need to.

The exciting ocular feature of this animal, though, is its third eye shown from the ventral aspect on the cover photograph (lower cover image). Found as a triplet lens system only in the males, this third eye is the "nauplier" eye. Nauplius means larval form, and most crustaceans have larval forms. In the nauplius form, these crustaceans make up part of the immense zooplankton of the ocean. Once hatched, most crustaceans proceed though six nauplier stages punctuated by a moult between each stage. The nauplier stage of many copepods has but one eye, the nauplier eye. In the males of Pontella scutifer, this nauplier eye is retained throughout life even as the animal goes on to develop two simple eyes, really just ocelli, on the dorsal aspect of the cephalosome.

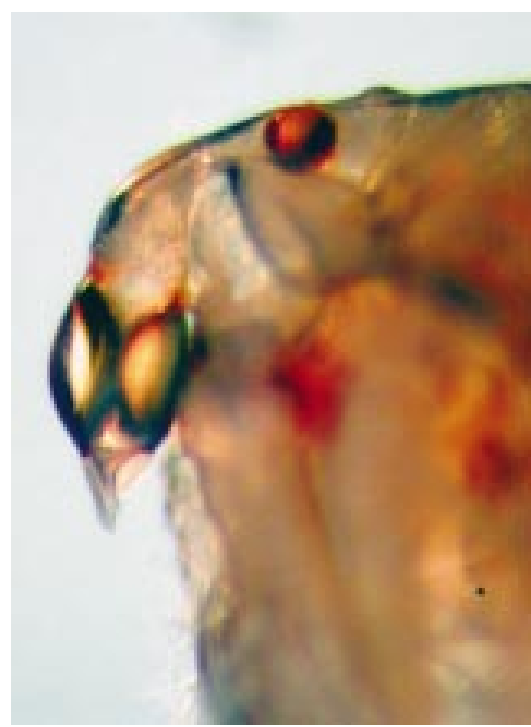

Sagittal view of head of Pontella. Note the parabolic nature of the lens when compared to cover photograph.

The nauplier eye of Pontella is extraordinary indeed. As can be seen from the cover, the nauplier eye contains three consecutive lenses with a total of six (!) photoreceptors behind the lens system. Each lens in this system is approximately $100 \mu \mathrm{m}$ and probably has an index of refraction of 1.52, according to Land and Nilsson in their book Animal Eyes (Oxford: Oxford University Press 2002:6970). Carotenoid pigment, appearing as red in the photographs, lines the eye cup behind the third lens. This condensing lens-like ornament still holds its secrets as it has not been completely evaluated optically or neurologically. Nevertheless, Land and Nilsson have evaluated the system and have determined that the front surface of the first lens, as can be seen from the photograph on the cover and in the figure on this page, is parabolic. This allows the triplet lens system to eliminate, or at least greatly reduce, spherical aberration. This aspheric lens is an alternative to the graded index of refraction lens found commonly in fish and discussed in the May 2002 BJO essay. Land and Nilsson, by the documentation of ray tracing, show that this unique lens system creates a single point image. Although we don't completely understand how this system works, it must relate to mating since the females have a doublet rather than a triplet lens system. And finding a female may be quite a challenge for these males, as they form massive swarms that are almost exclusively male with a small number of females. This probably creates intense competition for mating, for the first task must be finding the females in these swarms. The females of this species are blue with yellow spots. The asymmetry of two of the photoreceptor elements, called rhabdomes, in the male nauplier eye suggests a centre surround detection system that would respond to the female colour pattern. The other two pairs of rhabdomes have reflecting pigment behind them and may receive a reflected and refocused image, as do some scallops. But this is just a guess. The truth may be stranger yet and is simply unknown.

Other sensory mechanisms are well developed in pelagic copepods and probably more important than the visual ones, but are likely to work cooperatively. The first antenna contains antennular mechanoreceptors and chemosensory setae which have exceptional sensitivity to movements and even sensitivity to high frequency sound. These receptors are probably most useful in distinguishing prey from predators and the determination of prospective mates. These receptors have surprisingly large nerves with a form of myelin to allow rapid transmission of the signal to the muscular system of its tail. These muscles can flex quickly providing surprisingly rapid locomotion and measured in a related species to a maximum speed of 500 body lengths per second. This may not seem so fast, but given the body size of the copepods, would be equivalent to a cheetah running at over 2000 miles an hour!

So, although small, this copepod carnivore holds visual secrets with an eye for the ladies.

I R Schwab irschwab@ucdavis.edu

Photographs by the author.

Thanks to Frank D Ferrari, T Chad Walter, and the Smithsonian National Museum of Natural History for loan of the specimens, and comments on the essay. 